\section{ELECTIVE SURGICAL MRSA SCREENING IN A PAEDIATRIC HOSPITAL}

\section{J. Room 1 , J. Gray²}

${ }^{1}$ Infection Prevention and Control, Birmingham Children's Hospital NHS Foundation Trust, ${ }^{2}$ Microbiology Department, Birmingham Children's Foundation NHS Trust, Birmingham, UK

UK Guidance on MRSA screening requires that 'high-risk' children are screened on admission to hospital. We investigated the prevalence of MRSA in elective non-day case paediatric surgical admissions to determine the prevalence of MRSA and therefore the possible value of screening.

We screened 2222 surgical children by nose swabs cultured on MRSA selective medium between January and September 2009 and found the following prevalences:

- $3 / 1236(0.24 \%)$ Short stay surgical patients

- $8 / 544(1.47 \%)$ Longer stay surgical patients

- $2 / 342(0.58 \%)$ Neurosurgical patients

- 0/100 Renal surgical patients

- Overall prevalence 13/2222 (0.59\%).

The prevalence of MRSA in short stay (1-2 days) surgical patients (mainly ENT patients) was very low. The nature of their surgery probably presents a low risk of post-operative infections. Their short hospital stay means that even if MRSA-positive, there would be a low risk of them being the source of patient-to-person transmission of MRSA. By contrast the prevalence of MRSA in longer stay patients was significantly higher $(p=0.002)$. These patients present a greater infection control hazard and would be at greater risk of infection. All of our cases received decolonisation treatment: none developed post-operative infections with MRSA whilst under hospital care, and there was no evidence of nosocomial transmission. We conclude that children requiring short hospital stays for straightforward surgery do not need screening for MRSA. However, we recommend that screening of longer stay surgical patients should be considered.

\section{PVL-PRODUCING US 300 MRSA OUTBREAK ON A PAEDIATRIC BURNS UNIT}

\author{
J. Room ${ }^{1}$, J. Gray'2, M. Patel' ${ }^{2}$, C. Thomas ${ }^{3}$
}

${ }^{1}$ Infection Prevention and Control Team,

${ }^{2}$ Microbiology Department, ${ }^{3}$ Burns Unit, Birmingham Children's Hospital NHS Foundation Trust, Birmingham, UK

The US 300 community-acquired MRSA strain remains relatively uncommon in Europe. We report on the successful management of an outbreak with such a strain on a paediatric Burns Unit affecting 3 patients, 2 staff members and 1 relative.

The outbreak was identified when 1 staff member and 2 children managed as outpatients presented with cutaneous abscesses on the same day. Because of the distinctive clinical presentation, screening of all staff and current inpatients was immediately instigated (ahead of laboratory identification of the outbreak strain): 1/90 staff and 0/6 patients carried the outbreak strain. Environmental sampling (100 sites) was also undertaken: only the playroom was contaminated. Outpatients were screened only if symptomatic.

One further outpatient and one family member of a case presented with cutaneous abscesses over the next 4 days. All isolates were confirmed to be a single PVL-producing strain by phage-typing and genotyping that was closely related to US 300 .

The following controls were put in place:

1. Parents and family doctors of all 74 patients managed during a three-week putative exposure period were contacted: no further clinical cases identified.

2. Enhanced cleaning of the Burns Unit, including whole room decontamination (Meditrox 100, Pershore, UK): follow-up environmental samples were negative.

3. Affected staff excluded from work until MRSA-negative.

4. Household members of confirmed cases managed according to national guidelines.

Two months after the last case, no more colonised or infected patients have been identified. Early and vigorous infection control interventions appear to have contained a potentially serious outbreak. 